\title{
Management of strabismus in thyroid eye disease
}

\begin{abstract}
Thyroid eye disease is an auto-immune condition characterised by an acute inflammatory phase followed by a fibrotic phase, which sometimes leads to restricted eye movements and diplopia. Medical treatment with systemic steroids with or without orbital radiotherapy and immunosuppression can control the inflammatory response. Strabismus surgery should be carried out only after the inflammation is no longer active and after any decompression surgery. Surgery comprises recession of tight muscles using adjustable sutures so as to maximise the area of binocular single vision. There is debate as to whether adjustable sutures should be used for the inferior rectus muscle. Patients should be encouraged to have realistic expectations, as binocular single vision may not be achievable in all directions of gaze and lid retraction may be made worse by surgery.
\end{abstract} Eye (2015) 29, 234-237; doi:10.1038/eye.2014.282; published online 19 December 2014

The Bristol Eye Hospital, Bristol, UK

\section{Correspondence:}

R Harrad, The Bristol Eye Hospital, Lower Maudlin Street, Bristol BS1 2LX, UK Tel: +44 (0)11 7342 4689; Fax: +44 (0)11 79284891 .

E-mail: r.a.harrad@

bristol.ac.uk

Received: 10 October 2014 Accepted: 13 October 2014 Published online: 19

December 2014

This work was presented at the Cambridge Ophthalmological Symposium.

\section{Pathogenesis}

Thyroid eye disease (TED) is an auto-immune condition, characterised by auto-antibodies to the thyroid stimulating hormone receptor and the insulin-like growth factor receptor. In the initial acute phase there is lymphocytic infiltration and oedema of the extra-ocular muscles with deposition of glycosaminoglycans and hyaluronic acid as well as adipogenesis. ${ }^{1}$ These processes can lead to an increase in the volume of the contents of the orbit with resultant proptosis and raised intra-orbital pressure. Later the inflammation subsides, but there may be fibrotic change in the orbit leading to tightness and restriction of movement of the extra-ocular muscles. The inferior rectus is most commonly affected, followed by the medial and superior rectus. ${ }^{2}$ Involvement of extra-ocular muscles may be bilateral or unilateral.

Sometimes only one muscle is affected. It is not known why some muscles are more commonly involved than others. The inferior rectus is the bulkiest and most tonically-active extra-ocular muscle; it may be that the degree of muscle activity and hence blood supply is a factor.

\section{Clinical assessment and non-surgical management}

The presence of lid-lag or lid retraction in an adult presenting with a recent onset of strabismus is highly suggestive of TED. Thyroid function tests are usually diagnostic, but a small proportion of patients with TED have normal thyroid function tests. Patients with TED are assessed for disease severity and activity, and treatment in the form of immunosuppression is given depending on the degree of disease activity according a clinical scoring system. ${ }^{3}$ Magnetic resonance imaging (MRI) scan of the orbits, particularly short tau inversion recovery ${ }^{4}$ sequence, is useful in gauging the disease activity, and demonstrating muscle involvement and optic nerve compression (Figure 1). Patients should be advised to stop smoking; smokers with TED are twice as likely to need strabismus surgery. ${ }^{5}$ Systemic steroids are prescribed with or without additional immunosuppression in the form of Azathiaprine or Cyclosporin or lowdose radiotherapy. Biological agents, such as Rituximab, are now sometimes employed and there is some evidence that cyclo-oxygenase inhibitors, such as Indomethacin, may be effective. ${ }^{6}$ Studies on medical treatment of TED have mostly been retrospective with small numbers and a tendency to include both active and inactive disease. Randomised control trials (RCTs) of radiotherapy have produced conflicting results. The CIRTED study, a multicentre double armed RCT of radiotherapy vs 
placebo, and Azathiaprine immunosuppression vs placebo, for patients on treatment with steroids for active TED is underway and will report the outcomes in 2015. It is widely observed that strabismus surgery is now carried out less frequently over recent years in TED patients, as a result of better medical management.

\section{Timing of surgery}

Surgery for strabismus in TED should not be carried out until the condition has become inactive and any orbital decompression has been performed. Patients who have already had a prolonged course of treatment and who are disabled by diplopia are understandably keen to have their strabismus corrected as soon as possible. However measurements of the strabismus should be stable for at least 6 months prior to surgery; one study has reported a significant change in the strabismus in $30 \%$ of patients after 6 months of stable measurements. ${ }^{8}$ Eyelid surgery should be delayed until after strabismus surgery. Some authors advocate the use of Botox in the acute phase, and prisms can be helpful if there is a small deviation.

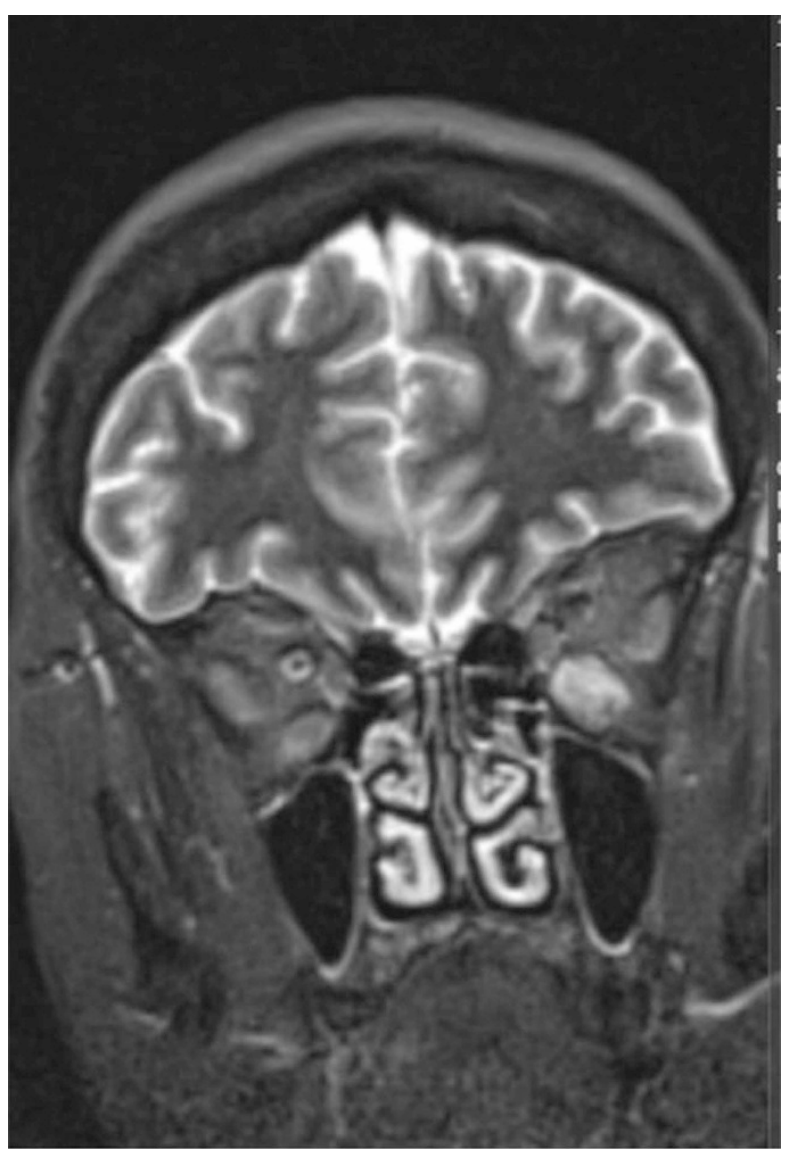

Figure 1 STIR MRI scan of the orbits showing enlarged and oedematous left inferior rectus muscle.

\section{The surgical plan}

At surgery the affected extra-ocular muscles are usually tight and fibrotic. Gaining access to the muscles can be challenging. Following decompression muscles may be incarcerated in bone. Where a deviation has increased significantly, following decompression, it advisable to repeat the MRI scan of the orbits. A 43-year-old man presented with a left esotropia following three wall orbital decompressions for proptosis and corneal exposure. There was 55 prism dioptres of esotropia and the left eye was fixed in adduction. MRI scan showed adhesion between the medial orbital wall and the medial rectus muscle along almost its entire length (Figure 2). At surgery it was impossible to free the medial rectus muscle, which was recessed $7 \mathrm{~mm}$ on an adjustable suture, using the relaxed-muscle technique and recessed a further $2 \mathrm{~mm}$ on adjustment. Three months later there was a good result with 5 prism dioptres of esotropia with binocular single vision in the primary position.

Surgery is carried out with the patient paralysed under general anaesthesia and an experienced assistant is recommended. A Fison's retractor is helpful to expose the muscle. Sometimes when the muscle is very tight it may be necessary to divide it using a scalpel while protecting the globe with the squint hook. A forced duction test should be carried out in order to assess the tightness of all the muscles. The position of the muscle insertion should be measured prior to dividing it and again after division. The insertion of a very tight muscle

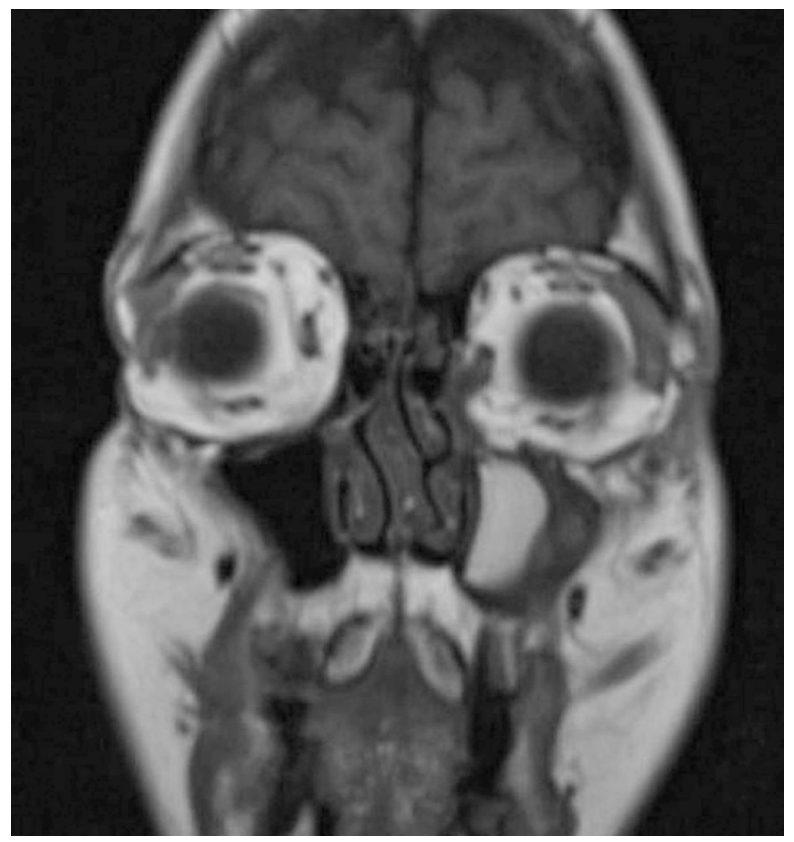

Figure 2 MRI scan of the orbits showing incarceration of the left medial rectus muscle in the medial orbital walling following orbital decompression. 
may move up to $2 \mathrm{~mm}$ anteriorly after division of the muscle, and unless this is recognised the muscle may be under-recessed. Surgery comprises recession of the tight muscles. Resection is rarely carried out. Some authors advocate fixing the recessed muscle in the 'relaxedmuscle position' $; 9$ this is where the muscle lies at surgery with the eye centrally placed. There is some controversy over the use of adjustable sutures when recessing the inferior rectus muscle. Several authors have described late over-correction using this technique. ${ }^{10,11}$ However, a recent study found no significant difference between fixed recession and adjustable sutures. ${ }^{12}$ Some surgeons use non-absorbable sutures to reduce the risk of late over-correction, but these sutures have a higher risk of infection and a tendency to erode through the conjunctiva. Kushner reported $100 \%$ success in abolishing muscle slippage by employing a semiadjustable suture technique. ${ }^{13}$ An alternative approach is to recess the inferior rectus using a fixed suture while recessing the contralateral superior-rectus muscle on an adjustable. This technique also has the advantage of reducing the amount of surgery on each of the vertical muscles thus limiting the amount of surgically-induced upper and lower lid retraction. There are no published RCTs comparing adjustable and non-adjustable sutures. Recession of an inferior rectus muscle results in between 3 and 4 prism dioptres of effect per mm of surgery. ${ }^{12}$ When recessing a vertical muscle, it should be sagittalised, that is moved in a nasal direction by half an insertion width in order to reduce the ' $\mathrm{A}$ ' or ' $\mathrm{V}$ ' pattern induced by weakening its adducting power. Recession of a vertical muscle carries the risk of exacerbating lid retraction as there are fibrous connections between the superior rectus and the levator complex in the upper lid, and the inferior rectus and the lower lid retractors. At the time of strabismus surgery it is advisable to divide these fibres as far posteriorly as possible. Conversely, recessing a tight inferior rectus may reduce the extent of ipsilateral upper lid retraction where this is owing to the Hering's law and secondary to increased tone in the superior rectus. Several authors advise aiming to under-correct a vertical deviation to allow for a late over-correction. ${ }^{12}$ The aim should be to achieve single vision with both eyes in about 10 degrees of downgaze. This facilitates reading and walking down steps while requiring only a minimal chin-up head position for distance vision. Where there is no excyclotortion in the presence of a tight inferior rectus muscle a tight superior oblique should be suspected and if present it will be tight on traction testing following division of the inferior rectus and per-operative measurement will show incyclotortion. The tight superior oblique should be recessed. ${ }^{14,15}$

Patients' expectations have to be carefully managed: it is usually possible to achieve single vision in the primary position with or without the aid of prisms in one operation, but the patient may have to get used to moving their head in order to have single vision in other directions. Sometimes more than one operation may be necessary to achieve a satisfactory outcome. Fortunately, many patients develop an impressively large fusion range which helps to reduce their diplopia. Patients need to be warned that their proptosis and lid retraction may become more noticeable after strabismus surgery: recession of 2 tight rectus muscles may lead an increase in proptosis of $2-3 \mathrm{~mm}$.

\section{Conclusion}

Strabismus surgery in TED should not be carried out until the disease is inactive and after any orbital decompression. Measurements should be stable for at least 6 months. Recession of those muscles that are tight on forced duction test using adjustable sutures brings about the best results. Although some authors report good results using this technique, the use of adjustable sutures on the inferior rectus is probably best avoided.

\section{Conflict of interest}

The author declares no conflict of interest.

\section{References}

1 Eckstein AK, Johnson KT, Thanos M, Esser J, Ludgate M. Current insights into the pathogenesis of Graves' orbitopathy. Horm Metab Res 2009; 41(6): 456-464.

2 Dyer JA. The oculorotary muscles in Graves' disease. Trans Am Ophthalmol Soc 1976; 74: 425-456.

3 Mourits MP, Prummel MF, Wiersinga WM, Koornneef L. Clinical activity score as a guide in the management of patients with Graves' ophthalmopathy. Clin Endocrinol (Oxf) 1997; 47(1): 9-14.

4 Laitt RD, Hoh B, Wakeley C, Kabala J, Harrad R, Potts M et al. The value of the short tau inversion recovery sequence in magnetic resonance imaging of thyroid eye disease. Br J Radiol 1994; 67(795): 244-247.

5 Rajendram R, Bunce C, Adams GG, Dayan CM, Rose GE. Smoking and strabismus surgery in patients with thyroid eye disease. Ophthalmology 2011; 118(12): 2493-2497.

6 Kuriyan AE, Woeller CF, O'Loughlin CW, Phipps RP, Feldon SE. Orbital fibroblasts from thyroid eye disease patients differ in proliferative and adipogenic responses depending on disease subtype. Invest Ophthalmol Vis Sci 2013; 54(12): 7370-7377.

7 Rajendram R, Lee RW, Potts MJ, Rose GE, Jain R, Olver JM. Protocol for the combined immunosuppression \& radiotherapy in thyroid eye disease (CIRTED) trial: a multi-centre, double-masked, factorial randomised controlled trial. Trials 2008; 9: 6.

8 Lee YH, Oh SY, Hwang JM. Is 6 months of stable angle of strabismus enough to perform surgery in patients with strabismus related to thyroid ophthalmopathy? Br J Ophthalmol 2010; 94(7): 955-956. 
9 Dal Canto AJ, Crowe S, Perry JD, Traboulsi EI. Intraoperative relaxed muscle positioning technique for strabismus repair in thyroid eye disease. Ophthalmology 2006; 113(12): 2324-2330.

10 Scott WE, Thalaker JA. Diagnosis and treatment of thyroid myopathy. Ophthalmology 1981; 88: 493-498.

11 Sprunger DT, Helveston EM. Progressive overcorrection after inferior rectus recession. J Pediatr Ophthalmol Strabismus 1993a; 30: 145-148.

12 Peragallo JH, Velez FG, Demer JL, Pineles SL. Postoperative drift in patients with thyroid ophthalmopathy undergoing unilateral inferior rectus muscle recession. Strabismus 2013; 21(1): 23-28.

13 Kushner BJ. An evaluation of the semiadjustable suture strabismus surgical procedure. J AAPOS 2004; 8: 481-487.

14 Thacker NM, Velez FG, Demer JL, Rosenbaum AL. Superior oblique muscle involvement in thyroid ophthalmopathy. J AAPOS 2005; 9(2): 174-178.

15 Holmes JM, Hatt SR, Bradley EA. Identifying masked superior oblique involvement in thyroid eye disease to avoid postoperative A-pattern exotropia and intorsion. I AAPOS 2012; 16(3): 280-285. 